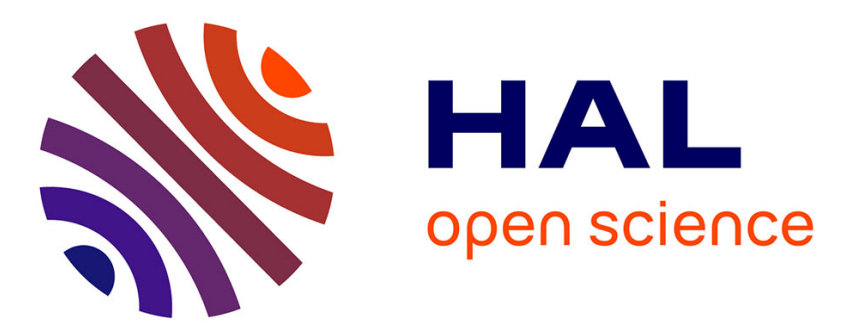

\title{
Comparison of absolute and relative strategies to encode sensorimotor transformations in tool-use
}

\author{
Raphael Braud, Alexandre Pitti, Philippe Gaussier
}

\section{To cite this version:}

Raphael Braud, Alexandre Pitti, Philippe Gaussier. Comparison of absolute and relative strategies to encode sensorimotor transformations in tool-use. IEEE International Conference on Development and Learning and on Epigenetic Robotics, Aug 2015, Providence, United States. pp.267-268, 10.1109/DEVLRN.2015.7346154 . hal-01267033

\section{HAL Id: hal-01267033 https://hal.science/hal-01267033}

Submitted on 19 Feb 2016

HAL is a multi-disciplinary open access archive for the deposit and dissemination of scientific research documents, whether they are published or not. The documents may come from teaching and research institutions in France or abroad, or from public or private research centers.
L'archive ouverte pluridisciplinaire HAL, est destinée au dépôt et à la diffusion de documents scientifiques de niveau recherche, publiés ou non, émanant des établissements d'enseignement et de recherche français ou étrangers, des laboratoires publics ou privés. 


\title{
Comparison of absolute and relative strategies to encode sensorimotor transformations in tool-use
}

\author{
Raphael Braud, Alexandre Pitti and Philippe Gaussier \\ Laboratory ETIS, UMR CNRS 8051 \\ University of Cergy-Pontoise, ENSEA, France \\ E-mail: raphael.braud@u-cergy.fr
}

\begin{abstract}
We explore different strategies to overcome the problem of sensorimotor transformation that babies face during development, especially in the case of tool-use. From a developmental perspective, we investigate a model based on absolute coordinate frames of reference, and another one based on relative coordinate frames of reference.

In a situation of sensorimotor learning and of adaptation to tool-use, we perform a computer simulation of a 4 degrees of freedom robot. We show that the relative coordinate strategy is the most rapid and robust to re-adapt the neural code.
\end{abstract}

\section{INTRODUCTION}

In order to use a tool, the brain has to adapt its body schema [1]. The role of the body schema is to encode our body in the brain, and the question is still open about how it is done, see [2].

Nonetheless, it is clear that in order to be fast, one has to ether adapt rapidly the model of the arm[1] or to learn a model of the tool [3]. We hypothesize that we need both: (i) a slight adaptation of the body schema when using a tool instead of a full re-learning of the arm's internal model, (ii) a learning of the relevant sensory context in which this adaptation occurs [4].

\section{Methods}

We present in fig. 1 the two main strategies for sensorimotor coordination that can achieve a reaching task, based either on an absolute strategy (AS) or a relative strategy (RS) for sensorimotor encoding between the motor coordinates $\boldsymbol{\theta}$ and the end-effector coordinates $\mathbf{X}$, see [5] for more details. The absolute strategy consists in learning the one-to-one mapping between spatial coordinates and motor configuration, see fig. 2 . The relative strategy consists in learning the one-to-one mapping between spatial directions of the end-effector and joint angles changes that cause movement in the spatial direction, see fig. 3.

Both strategies encode sensory inputs through an ARTlike categorization layer called a Selective Adaptive Winner (SAW), and map input contexts to output predictions in a supervised fashion using a Least Mean Square (LMS) rule. In the absolute strategy, associations are learned between sensor categories and motor activities. Sensors are encoded through a SAW, and then associated to motor activities using a LMS rule. In contrast, the relative strategy predicts spatial movements using a Sensorimotor Law Encoder (SLE). The SLE selects the most appropriate sensory category using a Winner Takes All

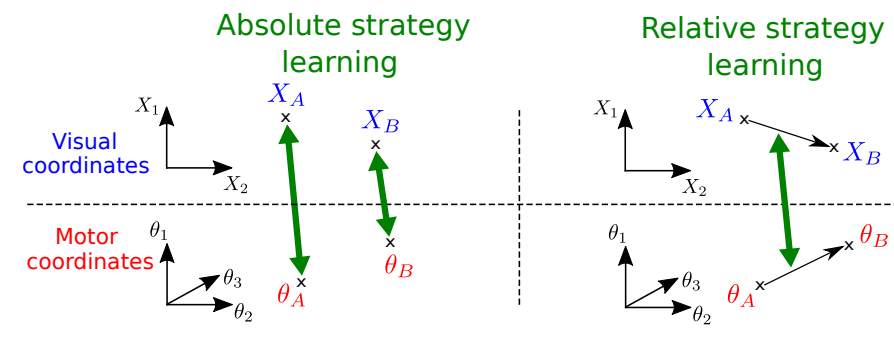

Fig. 1. Absolute versus relative sensorimotor encoding

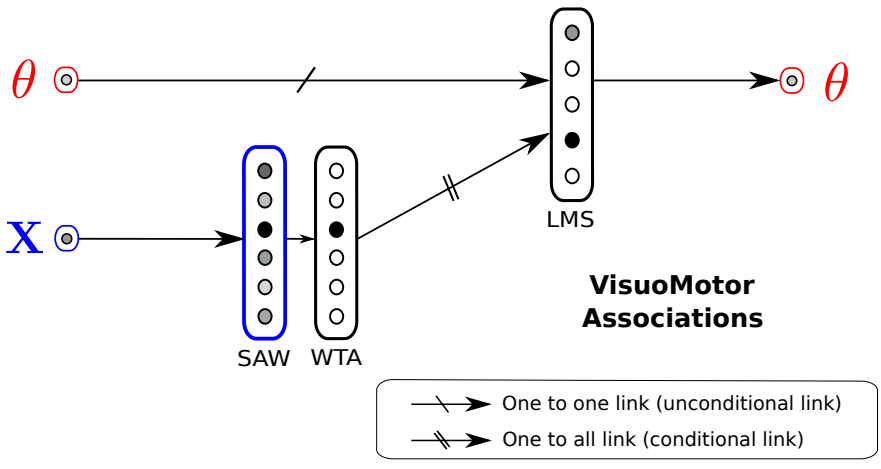

Fig. 2. Absolute visuomotor associative model based on classical conditioning. Sensors are encoded through a SAW, and then associated to motor activities using a LMS rule.

(WTA) neural network. Then, it multiplies the corresponding sensory pattern by the scalar motor activity $\dot{\theta}_{i}$. In the learning phase, the real sensory variation $\dot{X}_{j}$ is mapped to the winner pattern using the LMS rule. In the use phase, this allows prediction of the sensory variation $\dot{X}_{j}^{\text {Predicted }}$ associated with the currently recognized sensory pattern modulated by the motor activity.

The absolute strategy, can be seen as a homeostatic system. After the learning of its visual categories, even in the presence of noise or changes, any conflict in its visual inputs will force the system to stabilize its output to an equilibrium point. Such a mechanism is used here to reach a given target, given a visual input. Nevertheless, those inputs could for example be built as visio-motor attractors with both vision and proprioceptive inputs, see [6], [7]. In the relative strategy, we used a mechanism based on an actor-critic paradigm with no learning and a critic based not on real actions but on simulated ones. 


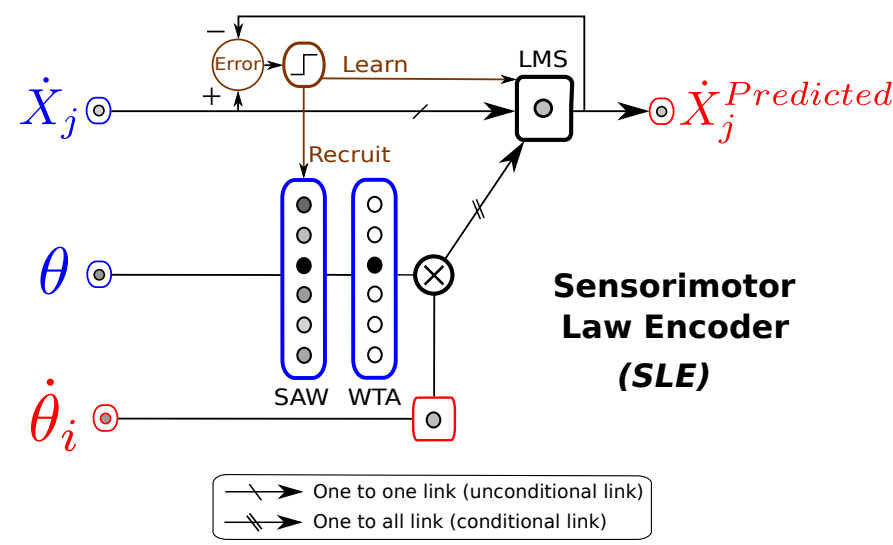

Fig. 3. Relative encoding using SLE. $\dot{X}_{j}$ is predicted, through a sensory categorization multiplied by a scalar corresponding to a motor activity $\dot{\theta}_{i}$, using a LMS rule.

\section{EXPERIMENTS}

a)
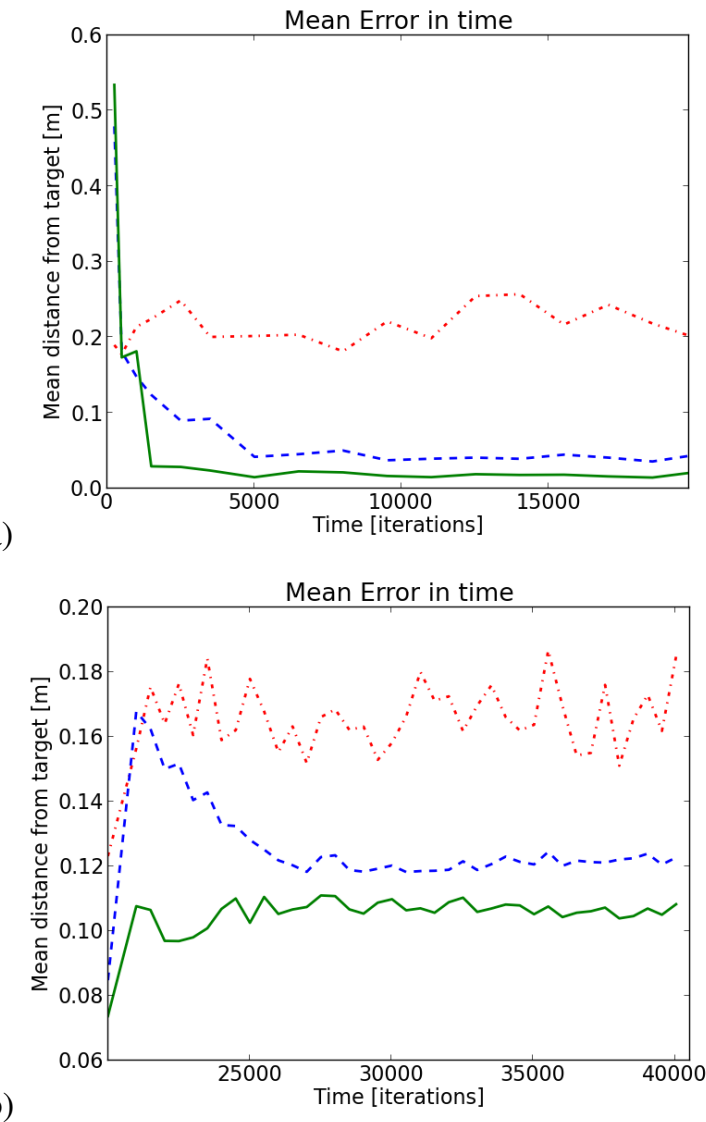

Fig. 4. In red and dotted for CS, in blue and dashed for AS and in green for the RS a) Mean error for the reaching task during development b) Mean error for the reaching task after learning, while the geometry of the robot changes (by modelling the addition of a tool).

We use a 4-DOF Katana in our experiment in 3D space to test the learning performance of the reaching task for the AS and RS models, and with a control strategy (CS) model, based on a reinforcement of rewarded actions, for comparison. The reaching task is a continuous sequence of target coordinates in $3 \mathrm{D}$ space the 3 models have to achieve.

The three strategies are performing a motor babbling and at regular periods we test each algorithm for reaching. In fig. 4 a), we plot the evolution of those values in time. We note that CS, in red and dotted, remains quite constant since it does not learn. The AS model, in blue, decreases rapidly and then converges slowly until the whole space has been explored. The RS model beside decreases faster than the AS model.

To test the robustness of each strategy, we add a tool to each model, which has the effect of increasing the length of the final segment. Results are shown in fig. 4 b).

Performances of the AS model indicate that it needs to readapt its body schema as during the babbling phase. The AS model has to learn every association again to adapt to the tool, whereas the RS model swiftly converges to the correct solution. Contrarily to the AS, the RS does not need to relearn its sensorimotor control to adapt its actions since it learns the kinematics and not absolute coordinates positions, and involves directions from the end-effector (the hand, then the tool) to the target instead of the final position desired.

\section{CONCLUSION}

In this paper, we have investigated how sensorimotor information is encoded with respect to an absolute strategy and to a relative strategy. We ask ourself how "contextual" information is encoded and what it codes for. Experiments with a simulated 4-DOF katana arm for AS, RS and CS models indicate a good robustness when a tool is added for the RS, whereas for $\mathrm{AS}$, the robot has to re-learn its whole visio-motor mapping. Considering monkey experiments showing the rapid adaptation to a tool, our results tend to make the RS more relevant for it. Moreover, it is possible that both AS and RS models can learn in parallel, while CS seems a good back-up solution in case of failure of the two other models. In future works, we seek to address the following questions : how the 3 strategies could be always present during development, and how they can develop complementary properties.

\section{REFERENCES}

[1] A. Maravita and A. Iriki, "Tools for the body (schema)," Trends in cognitive sciences, vol. 8, no. 2, pp. 79-86, 2004.

[2] M. Hoffmann, H. G. Marques, A. Hernandez Arieta, H. Sumioka, M. Lungarella, and R. Pfeifer, "Body schema in robotics: a review," Autonomous Mental Development, IEEE Transactions on, vol. 2, no. 4, pp. 304-324, 2010.

[3] J. Kluzik, J. Diedrichsen, R. Shadmehr, and A. J. Bastian, "Reach adaptation: what determines whether we learn an internal model of the tool or adapt the model of our arm?" Journal of neurophysiology, vol. 100, no. 3, pp. 1455-1464, 2008.

[4] N. Cothros, J. Wong, and P. Gribble, "Are there distinct neural representations of object and limb dynamics?" Experimental Brain Research, vol. 173, no. 4, pp. 689-697, 2006.

[5] D. Bullock, S. Grossberg, and F. H. Guenther, "A self-organizing neural model of motor equivalent reaching and tool use by a multijoint arm," Journal of Cognitive Neuroscience, vol. 5, no. 4, pp. 408-435, 1993.

[6] P. Gaussier and S. Zrehen, "Perac: A neural architecture to control artificial animals," Robotics and Autonomous Systems, vol. 16, no. 2, pp. 291-320, 1995.

[7] A. de Rengervé, P. Andry, and P. Gaussier, "Online learning and control of attraction basins for the development of sensorimotor control strategies," Biological cybernetics, vol. 109, no. 2, pp. 255-274, 2015. 